
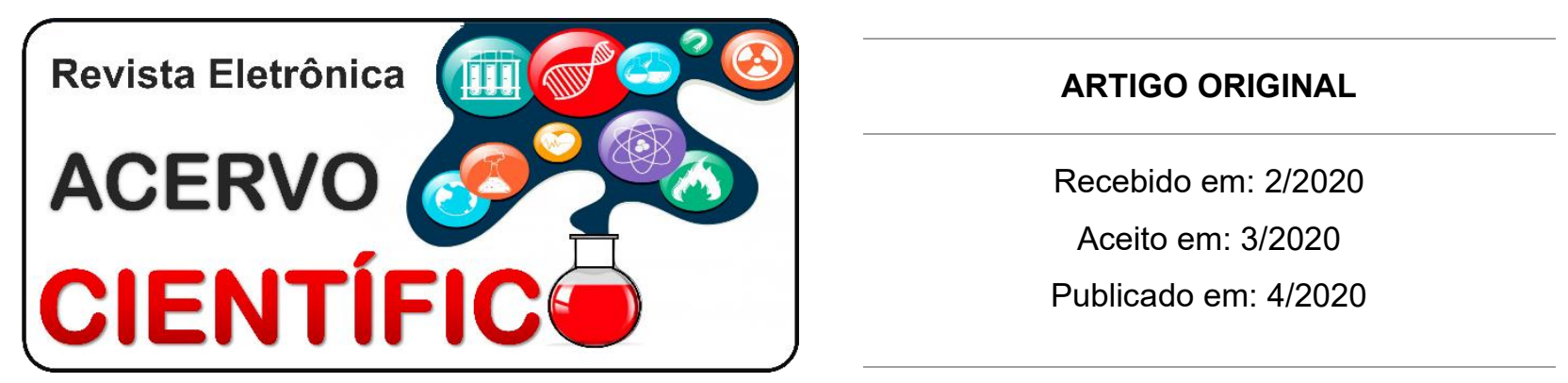

Recebido em: 2/2020

Aceito em: $3 / 2020$

Publicado em: 4/2020

\title{
Fobia social e a autopercepção do desempenho de estudantes de medicina submetidos à metodologia ativa em Sergipe
}

Social phobia and self-perception performance of medical students submitted to an active methodology in Sergipe

Fobia social y la auto percepción del rendimiento de estudiantes de medicina enviados a la metodología activa en Sergipe

Kamila Maria de Andrade Santos Silveira ${ }^{1 *}$, Mayana Lula Andrade ${ }^{1}$, Eusébio Lino dos Santos Júnior $^{1}$, Paulo Milhomem Ferro Neto ${ }^{2}$, Déborah Pimentel ${ }^{1,3}$.

Resumo: Esse artigo buscou investigar a influência dos sintomas de Fobia Social (FS) na autopercepção do desempenho acadêmico dos estudantes de Medicina submetidos ao método Aprendizagem Baseada em Problemas (ABP). Trata-se de um estudo clínico, não experimental, exploratório, transversal, descritivo e de abordagem analítica quantitativa. No quesito de autopercepção do desempenho acadêmico, a maioria dos alunos tanto do grupo com FS (74\%) quanto do grupo sem FS $(86,4 \%)$, indicou seu desempenho como excelente, muito bom ou bom. Quanto a vontade de desistir do curso muitas vezes ou sempre, o percentual foi de 2,4\% nos estudantes sem FS, e 6,6\% entre aqueles com FS. Concluiu-se que o absenteísmo relacionado a sintomas ansiosos foi relativamente mais frequente em estudantes com sintomas de FS. Ademais, os estudantes com sintomas de FS tendem a avaliar seu desempenho como pior em comparação aos estudantes sem FS, além de demonstrarem mais dificuldades na realização das tarefas.

Palavras-chave: Estudantes de medicina, Rendimento acadêmico, Fobia social, Aprendizagem baseada em problemas.

Abstract: This article sought to investigate the influence of social phobia symptoms (SP) on the selfperception regarding academic performance of medical students undergoing the Problem-Based Learning (PBL) method. This is a non-experimental, exploratory, cross-sectional, descriptive and quantitative analytical study. Regarding self-perception of academic performance, most students from both the SP (74\%) and nonSP (86.4\%) groups indicated their performance as excellent, very good or good. As for the willingness to drop out of the course many times or always, the percentage was $2.4 \%$ in students without FS, and $6.6 \%$ among those with FS. It was concluded that absenteeism related to anxiety symptoms was relatively more frequent in

\footnotetext{
${ }^{1}$ Universidade Tiradentes (UNIT), Aracaju - Sergipe. *E-mail: kamilasilveira.92@gmail.com

2 Universidade Federal de Sergipe (UFS), Lagarto - Sergipe.

3 Universidade Federal de Sergipe (UFS), Aracaju - Sergipe.
} 
students with symptoms of FS. Furthermore, students with symptoms of SF tend to assess their performance as worse compared to students without SF, in addition to showing more difficulties in performing tasks.

Keywords: Medical student, Academic success, Social phobia, Problem-based learning.

Resumen: Este artículo buscaba investigar la influencia de los síntomas de la fobia social (FS) en la autopercepción del rendimiento académico de los estudiantes de medicina sometidos al método de aprendizaje basado en problemas (PBL). Este es un estudio clínico, no experimental, exploratorio, transversal, descriptivo con un enfoque analítico cuantitativo. Con respecto al rendimiento académico autopercibido, la mayoría de los estudiantes tanto en el grupo con FS (74\%) como en el grupo sin FS (86.4\%), indicaron que su desempeño era excelente, muy bueno o bueno. En cuanto al deseo de abandonar el curso muchas veces o siempre, el porcentaje fue de $2.4 \%$ en estudiantes sin FS y $6.6 \%$ entre aquellos con FS. Se concluyó que el absentismo relacionado con síntomas de ansiedad fue relativamente más frecuente en estudiantes con síntomas de FS. Además, los estudiantes con síntomas de FS tienden a evaluar su desempeño como peor en comparación con los estudiantes sin FS, además de mostrar más dificultades para realizar tareas.

Palabras clave: Estudiantes de medicina, Rendimiento académico, Fobia social, Aprendizaje basado en problemas.

\section{INTRODUÇÃO}

O sucesso está relacionado com a capacidade que o indivíduo tem de atender às expectativas sociais. No meio acadêmico, os alunos mais aptos a corresponder a essas demandas acabam se destacando entre os colegas e os professores, o que fomenta um ambiente competitivo e estressante. Ademais, essas exigências são acentuadas na medida em que os universitários são submetidos a avaliações constantes quanto a sua capacidade e desenvoltura. No intuito de garantir o sucesso profissional futuro, esse grupo se sente compelido a mostrar um desenvolvimento excepcional (ALMEIDA AM, et al., 2007; OLIVEIRA MA e DUARTE AMM, 2004).

Não obstante, no ambiente universitário há uma grande demanda a respeito das interações sociais por parte dos alunos, como acontece nos seminários, nas sessões tutoriais e ao esclarecer dúvidas com professores ou colegas. Essas circunstâncias podem levar alguns estudantes a se sentirem envergonhados ou humilhados diante das outras pessoas devido a sua performance, o que pode gerar enorme desconforto, sintomas ansiosos e a utilização de subterfúgios para escapar destas situações sociais (REGIS JMO, 2015; WAGNER MF, et al., 2014; PEREIRA SM e LOURENÇO LM, 2012; WONG N, et al., 2012; BURSTEIN M, et al., 2011; HEISER N, et al., 2009; FEHM L, et al., 2005).

Em meio a isso, os estudantes de Medicina, por sua vez, sofrem pressões específicas do seu curso e se tornam mais vulneráveis ao adoecimento mental, ao uso abusivo de substâncias químicas e ao suicídio quando comparados à população geral. Dentre os eventos que prejudicam a saúde mental desses alunos, incluem-se: o concorrido vestibular para acessar o curso; carga horária excessiva; grande quantidade de conteúdo a ser estudado; falta de tempo para o autocuidado e para desenvolver interações sociais fora do ambiente acadêmico; entrar em contato com o sofrimento humano e a morte e lidar com as exigências pessoais e sociais (AMORIM BB, et al., 2018; TENÓRIO LP, et al., 2016; MARAFANTII, et al., 2013; COSTA EFO, et al., 2010; ALMEIDA AM, et al., 2007).

Nos últimos tempos, as escolas médicas nacionais têm modificado as práticas de ensino-aprendizado com o uso das chamadas metodologias ativas. O intuito dessa mudança é favorecer que o perfil do egresso seja de um profissional com conhecimentos integrados a respeito do ser humano dentro de suas necessidades físicas, psíquicas e sociais, tendo um olhar mais humanizado e não meramente técnico. Dentre as metodologias ativas, destaca-se a Aprendizagem Baseada em Problemas (ABP), na qual os alunos são apresentados a uma situação-problema e são estimulados a refletir a respeito do caso apresentado, para que, então, sistematizem o seu próprio estudo na busca de conhecimentos que respondam ao desafio. Nesse 
contexto, o professor deixa o papel de gerenciador, e assume a postura de facilitador na busca do conhecimento por parte dos alunos. A ABP usa como base as chamadas sessões tutoriais, nas quais um grupo limitado de alunos se reúne com um tutor (professor) e desenvolvem discussões acerca da situaçãoproblema (TENÓRIO LP, et al., 2016; VIGNOCHI CM, et al., 2009).

Em meio a essa exposição, essas pessoas podem se tornar mais propensas a desenvolver algum tipo de transtorno de ansiedade, entre eles o transtorno de ansiedade social (TAS), ou fobia social (FS). A FS é um problema psiquiátrico frequente e de curso crônico, relacionado à manifestação de sintomas ansiosos mediante a exposição do indivíduo a uma situação social que, em geral, qualifique o seu desempenho, levando a um comprometimento da sua qualidade de vida e da sua funcionalidade, e, nos casos mais graves, pode ser debilitante e causar um prejuízo pessoal, profissional e acadêmico (REGIS JMO, 2015; WAGNER MF, et al., 2014; PEREIRA AS, et al., 2014; PEREIRA SM e LOURENÇO LM, 2012; BEIDEL DC, et al., 2010).

Sendo os sintomas de transtorno de ansiedade social uma condição prevalente e que compromente o desempenho do indivíduo, seria possível encontrar alunos com o transtorno tendo seu rendimento acadêmico afetado e com provável evasão do curso devido aos seus sintomas, principalmente dentro de uma metodologia de ensino que exige exposição. O presente estudo objetiva investigar a influência dos sintomas da fobia social na autopercepção dos estudantes de Medicina submetidos ao método Aprendizagem Baseada em Problemas acerca do desempenho acadêmico.

\section{MÉTODOS}

Trata-se de um estudo clínico, não experimental, exploratório, transversal, descritivo e de abordagem analítica quantitativa. É válido ressaltar que o presente trabalho se trata de um recorte feito de um estudo maior que objetivou estabelecer a prevalência dos sintomas da fobia social em estudantes de Medicina, submetidos ao método de ensino Aprendizagem Baseada em Problemas, no estado de Sergipe. Foram incluídos na pesquisa estudantes acima de 18 anos, de ambos os sexos, cujas instituições de ensino adotem o método $A B P$, e que estejam cursando quaisquer das etapas do $1^{\circ}$ ao $8^{\circ}$ semestre, que assinaram o termo de consentimento livre e esclarecido (TCLE), de acordo com as normas do Conselho Nacional de Saúde do Ministério da Saúde explicitadas na resolução no 466/12, e o trabalho somente foi iniciado após aprovação do Comitê de Ética em Pesquisa da Universidade Tiradentes (CEP/UNIT), com o parecer CAAE no 67748317.4.0000.5371.

A amostra populacional almejada foi baseada na fórmula de Gil (1999) para populações finitas, que analisa o tamanho necessário desta. Considerando 1600 alunos no total matriculados nestas etapas (800 em cada escola/universidade), com erro amostral de 3,2\% e com um nível de confiança de $95 \%$, considerando a prevalência de Fobia Social de 11,6\%, baseada em Batista et al., totalizando um mínimo de 322 estudantes.

Utilizou-se a amostra de 323 alunos, os quais responderam a um questionário contendo a Escala de Ansiedade Social de Liebowitz e mais outras perguntas elaboradas pelos próprios autores. Para incrementar a análise qualitativa dos resultados, foram acrescidas as seguintes perguntas: quantos dias de aula o aluno perdeu no último mês por medo, ansiedade e/ou evitação; como estava a capacidade de realizar as atividades acadêmicas no último mês; como o estudante avalia o seu rendimento acadêmico; se se sente envergonhado do rendimento acadêmico; se apresenta a sensação de humilhação ou vergonha quando é criticado quanto ao desempenho acadêmico; e se apresenta pensamentos de desistir do curso. Foi realizada, então, uma análise descritiva caracterizando as variáveis categóricas por meio de frequência absoluta e relativa. Para avaliar as associações entre variáveis categóricas foi utilizado o teste Qui-Quadrado de Pearson. O nível de significância adotado foi de 5\% e o software utilizado foi o R Core Team 2018.

\section{RESULTADOS}

O presente trabalho demonstrou que, dos 323 estudantes presentes na amostra, 30,8\% apresentaram Fobia Social em algum grau. Alguns aspectos sócio-demográficos foram analisados e comparados entre estudantes de medicina com e sem a Fobia Social, mostrando prevalência do transtorno na idade entre 18- 
25 anos, no sexo feminino, nos solteiros, na instituição privada e nos primeiros 2 anos de faculdade. O perfil sócio-demográfico dos entrevistados está representado na Tabela 1.

Tabela 1 - Características sociodemográficas da população com fobia social.

\begin{tabular}{lcccc}
\hline \multirow{2}{*}{ Característica } & Sem fobia social & Com fobia social & Ausentes & \multirow{2}{*}{ p-valor } \\
\cline { 2 - 3 } Idade (anos) & $\mathbf{n ~ ( \% )}$ & $\mathbf{n}(\%)$ & $\mathbf{N}(\%)$ & \\
\hline $18-25$ & $181(87,9)$ & $85(92,4)$ & $25(7,7)$ & 0,491 \\
$26-33$ & $20(9,7)$ & $5(5,4)$ & & \\
Acima de 33 & $5(2,4)$ & $2(2,2)$ & & \\
\hline Sexo & & & & \\
\hline Feminino & $102(49,3)$ & $73(79,3)$ & $24(7,4)$ & $<0,001$ \\
Masculino & $105(50,7)$ & $19(20,7)$ & & \\
\hline Estado Civil & & & & \\
\hline Solteiro & $191(94,1)$ & $88(97,8)$ & & \\
Casados/União estável & $12(5,9)$ & $2(2,2)$ & & \\
\hline Instituição & & & & \\
\hline Pública & $80(38,6)$ & $24(7,3)$ & \\
Privada & $127(61,4)$ & $67(72,9)$ & & \\
\hline Ano & & & & \\
\hline 1-2 & $136(65,7)$ & $62(67,4)$ & & \\
3-4 & $75(36,2)$ & $30(32,6)$ & & \\
\hline
\end{tabular}

Legenda: $n$ - Frequência absoluta. \% - Frequência relativa percentual. Teste Qui-Quadrado de Pearson. Fonte: Silveira KMAS, et al., 2020.

Esta análise revelou que o grupo de indivíduos com fobia social apresentou maior índice de absenteísmo por medo, ansiedade ou evitação em comparação ao grupo sem fobia social $(p<0,001)$. Um percentual maior de indivíduos no grupo com fobia social $(34,8 \%)$ respondeu ter perdido "poucos dias de aula" por medo, ansiedade ou evitação em comparação ao grupo sem fobia social (14\%). Embora nenhum dos estudantes com fobia social tenha respondido que faltou a "metade dos dias de aula", um percentual maior de estudantes sem fobia social respondeu não ter perdido nenhum dia de aula em comparação ao grupo com fobia social (84,5\% e 65,2\%, respectivamente). Os resultados estão expressos na Tabela 2.

Tabela 2 - Perda de dias de aula no último mês, por medo, ansiedade ou evitação.

\begin{tabular}{lcccc}
\hline \multirow{2}{*}{ Respostas } & Sem fobia social & Com fobia social & \multirow{2}{*}{ p-valor } & Ausentes \\
\cline { 2 - 3 } & $\mathbf{n}(\%)$ & $\mathbf{n}(\%)$ & $\mathbf{n}(\%)$ \\
\hline Nenhum dia & $175(84,5)$ & $60(65,2)$ & $<0,001$ & $24(7,4)$ \\
\hline Poucos dias & $29(14,0)$ & $32(34,8)$ & & \\
\hline Metade dos dias & $3(1,4)$ & $0(0,0)$ & & \\
\hline Total & $207(100)$ & $92(100)$ & & \\
\hline
\end{tabular}

Legenda: $\mathrm{n}$ - Frequência absoluta. \% - Frequência relativa percentual. Teste Qui-Quadrado de Pearson. Fonte: Silveira KMAS, et al., 2020.

Em relação à autopercepção do desempenho acadêmico, a maioria dos alunos tanto do grupo com fobia social $(74 \%)$ quanto do grupo sem fobia social $(86,4 \%)$, indicou seu desempenho como excelente, muito bom ou bom; porém é possível perceber que o grupo com fobia social apresentou percentuais menores nesse quesito $(p=0,030)$. Os alunos com fobia social acabaram tendo um percentual maior de autoavaliação do seu desempenho como regular ou insuficiente. 
Ademais, quando se avaliou a capacidade de realizar as atividades acadêmicas, um maior índice de pessoas do grupo com FS, em relação às pessoas sem fobia social, afirmou ter necessitado de ajuda ou ter não ter feito às tarefas de forma adequada na metade do tempo, ou ainda ter feito as tarefas inadequadamente na maior parte do tempo $(p<0,001)$. A Tabela 3 informa o resultado quanto à capacidade dos alunos de realizar as atividades acadêmicas e a auto-avaliação dos estudantes a respeito do seu rendimento acadêmico.

Tabela 3 - Capacidade de realizar tarefas acadêmicas no último mês e avaliação do rendimento acadêmico no último mês.

\begin{tabular}{|c|c|c|c|c|}
\hline & \multirow{2}{*}{$\frac{\text { Sem fobia social }}{\mathrm{n}(\%)}$} & \multirow{2}{*}{$\begin{array}{c}\text { Com fobia social } \\
\mathrm{n}(\%)\end{array}$} & \multirow{2}{*}{ p-valor } & \multirow{2}{*}{$\begin{array}{c}\text { Ausentes } \\
\mathrm{n}(\%)\end{array}$} \\
\hline & & & & \\
\hline \multicolumn{5}{|l|}{$\begin{array}{l}\text { Capacidade de realizar tarefas } \\
\text { acadêmicas no último mês }\end{array}$} \\
\hline Muito bem & $78(37,7)$ & $17(18,5)$ & $<0,001$ & $24(7,4)$ \\
\hline Fez com pequenas dificuldades & $105(50,7)$ & $48(52,2)$ & & \\
\hline $\begin{array}{l}\text { Precisou de ajuda e/ou não fez } \\
\text { cerca da metade do tempo }\end{array}$ & $13(6,3)$ & $15(16,3)$ & & \\
\hline $\begin{array}{l}\text { Fez de maneira inadequada na } \\
\text { maior parte do tempo }\end{array}$ & $11(5,3)$ & $12(13,0)$ & & \\
\hline Total & $207(100)$ & $92(100)$ & & \\
\hline \multicolumn{5}{|l|}{ Rendimento acadêmico } \\
\hline Excelente, muito bom e bom & $178(86,4)$ & $68(74,0)$ & 0,030 & $25(7,7)$ \\
\hline Regular & $23(11,2)$ & $20(21,7)$ & & \\
\hline Insuficiente & $5(2,4)$ & $4(4,3)$ & & \\
\hline Total & $206(100)$ & $92(100)$ & & \\
\hline
\end{tabular}

Legenda: $\mathrm{n}$ - Frequência absoluta. \% - Frequência relativa percentual. Teste Qui-Quadrado de Pearson. Fonte: Silveira KMAS, et al., 2020.

Conforme os nossos resultados, os estudantes do grupo com fobia social exibem maior percentual no quesito "sentir vergonha do seu rendimento acadêmico na metade ou na maior parte do tempo no último mês" em relação ao grupo sem fobia social, $23,9 \%$ e $6,8 \%(p<0,001)$ respectivamente. Ademais, no grupo com fobia social há um discreto aumento em relação ao grupo sem fobia social em "sentir vergonha o tempo todo de seu rendimento acadêmico no último mês", ainda que em ambos os grupos esse percentual fosse baixo ( $0,5 \%$ no grupo sem fobia social, e $2 \%$ no grupo com fobia social).

Não obstante, com relação à sensação de humilhação ou vergonha quando se é criticado o desempenho acadêmico, houve uma diferença expressiva entre ambos os grupos, na qual os estudantes com fobia social informaram sentir-se dessa forma algumas vezes $(33,7 \%$, sendo que nos indivíduos sem fobia social é de $23,8 \%$ ) ou sempre (no qual $28,3 \%$ informaram se sentir envergonhados ou humilhados, em comparação aos $4,8 \%$ do grupo sem fobia social) ( $p<0,001)$.

Por fim, os alunos foram questionados a respeito do pensamento de desistir do curso. A maioria dos indivíduos sem FS $(71,2 \%)$ afirmou nunca ter pensado em desistir do curso, e, entre aqueles com FS, quase a metade nunca pensou em desistir do curso. Dos indivíduos sem FS, 25,8\% afirmaram vontade de desistir do curso uma ou algumas vezes, enquanto essa vontade foi manifestada em $43,9 \%$ dos que apresentam transtorno de ansiedade social. Um número pequeno de alunos afirmou vontade de desistir do curso muitas vezes ou sempre, sendo que dos estudantes sem FS apenas 2,4\% fez essa afirmativa, e 6,6\% entre aqueles com TAS $(p<0,001)$. Os resultados estão descritos na Tabela 4. 
Tabela 4 - Pensamento de desistir do curso.

\begin{tabular}{lccccc}
\hline & & Sem fobia social & Com fobia social & \multirow{2}{*}{ p-valor } & Ausentes \\
\cline { 2 - 3 } & & $\mathbf{n}(\%)$ & $\mathbf{n}(\%)$ & & \\
\hline $\begin{array}{l}\text { Pensou }(\%) \\
\text { curso }\end{array}$ & & & \\
\hline Nunca & $148(71,8)$ & $45(49,5)$ & \\
Uma ou algumas vezes & $53(25,8)$ & $30(43,9)$ & $<0,001$ & $26(8,0)$ \\
Muitas vezes ou sempre & $5(2,4)$ & $6(6,6)$ & & \\
\hline Total & $206(100)$ & $91(100)$ &
\end{tabular}

Legenda: $n$ - Frequência absoluta. \% - Frequência relativa percentual. Teste Qui-Quadrado de Pearson.

Fonte: Silveira KMAS, et al., 2020.

\section{DISCUSSÃO}

Aproximadamente um terço dos indivíduos $(30,8 \%)$ de nossa amostra pôde ser encaixado em algum grau de FS, semelhante ao resultado descrito por Regis JMO (2015) em um estudo realizado com estudantes de Medicina de uma universidade de São Paulo, no qual a prevalência de provável FS foi de $36,3 \%$. Nossos dados divergem da prevalência de FS em estudantes universitários brasileiros encontrada por Batista CA, et al. (2012) que foi de $11,6 \%$, valor semelhante foi encontrado na população brasileira e em universitários de outros países. É válido ressaltar que a amostra desse último autor não foi apenas de estudantes de Medicina, podendo-se inferir que os sintomas de FS podem ser mais prevalentes nesses estudantes que em outras populações. Divergências metodológicas também podem explicar essa incongruência nos dados, sendo que no trabalho de por Batista CA, et al. (2012), como já citado, a amostra não envolveu apenas estudantes de Medicina, e a pesquisa foi estruturada em duas fases, sendo que na primeira foi utilizado o Mini-Inventário de Fobia Social (Mini-SPIN) para se realizar a triagem dos alunos com sintomas de fobia social para se fazer posteriormente a aplicação, via telefone, da Entrevista Clínica Estruturada para o DSM-IV (SCID-I/P 2.0) para se confirmar o diagnóstico de FS.

Costa EFO, et al. (2010), ao pesquisar transtornos mentais comuns (TMC) em estudantes de Medicina de uma universidade sergipana, constatou que a maioria deles, mesmo aqueles com TMC, avaliaram como excelente ou bom o seu desempenho acadêmico $(81,7 \%)$, corroborando com os nossos dados. Ainda segundo esse estudo, o baixo desempenho acadêmico é um fator que indica aumento no risco de TMC, sendo que essas duas condições podem se retroalimentar. Devido às dificuldades associadas à FS, como a de falar em público, o desempenho acadêmico dessas pessoas pode ser prejudicado, inclusive levando a menores notas nas provas acadêmicas (REGIS JMO, 2015; BAPTISTA CA, et al., 2012). Além do receio de falar em público, outro grande medo das pessoas com FS é ficarem assustadas ao serem criticadas (REGIS JMO, 2015). Essas dificuldades podem se sobressaltar em estudantes com FS submetidos a metodologias ativas, como a $\mathrm{ABP}$, na qual os alunos precisam falar em público, nas sessões tutoriais, e ter seu desempenho avaliado para a aquisição de nota.

Um estudo realizado por Marafanti I, et al. (2013) averiguou o desempenho de estudantes de Medicina com sintomas de ansiedade, e constatou que a ansiedade não foi preditora de performance. A explicação, segundo Marafanti I, et al. (2013), é que o indivíduo pode lançar mão de um conjunto de estratégias cognitivas e comportamentais para lidar com as exigências internas e externas que são avaliadas como excessivas, e isso recebe o nome de coping. Talvez por isso, no nosso estudo, a maioria dos estudantes do grupo com fobia social avaliem seu rendimento como excelente, muito bom ou bom.

Não foram encontrados dados na literatura que corroborem com os achados de absenteísmo em dias de aula e de provas. Não obstante, é válido ressaltar que as pessoas com FS costumam buscar subterfúgios para se esquivar de situações que lhes causem desconforto, o que pode predispor os estudantes com FS à evasão do curso, sobretudo nos indivíduos com manifestações mais graves da doença (Batista CA, et al., 2012; PEREIRA SM e LOURENÇO LM, 2012). 
Os indivíduos com fobia social, portanto, acabam tendo menos escolaridade pelos altos índices de evasão escolar/acadêmica, o que corrobora com os resultados encontrados na nossa pesquisa, nos quais os alunos com FS manifestam, relativamente, maior vontade de desistir do curso (REGIS JMO, 2015; Batista CA, et al., 2012; PEREIRA SM e LOURENÇO LM, 2012; COSTA EFO, et al., 2010; ENG W, et al., 2005; OLIVEIRA MA e DUARTE AMM, 2004; BRUNELLO N, et al., 2000).

O presente estudo apresentou como limitação não abranger estudantes de medicina do método de aprendizagem tradicional, para que assim houvesse um grupo controle no comparativo com a metodologia de ensino Aprendizagem Baseada em Problemas. A quantidade de estudos sobre o rendimento acadêmico em estudantes de medicina com fobia social demonstrou-se limitado. Pretendemos, assim, estimular que novas pesquisas sejam realizadas nessa área.

\section{CONCLUSÃO}

Nesta pesquisa, o absenteísmo relacionado a sintomas ansiosos foi relativamente mais frequente em estudantes com sintomas de Fobia social. Ademais, os estudantes com sintomas de Fobia Social tendem a avaliar seu desempenho como pior em comparação aos estudantes sem Fobia Social, além de demonstrarem mais dificuldades na realização das tarefas. É de suma importância que as escolas médicas estejam preparadas para identificar e dar o correto encaminhamento para os estudantes que Medicina que expressam algum grau de adoecimento mental, haja vista que estes indivíduos são mais predispostos que a população geral a desenvolverem algum tipo de TMC. Os professores do método Aprendizagem Baseada em Problemas, por terem um contato mais próximo com estes alunos, devido ao número reduzido de pessoas nas sessões tutoriais, deveriam ser treinados para identificar déficits psicopedagógicos nestes alunos. O correto suporte psicopedagógico para um aluno com Fobia Social pode ajudá-lo a desenvolver melhor suas habilidades e qualidade de vida.

\section{AGRADECIMENTOS E FINANCIAMENTO}

Agradecemos aos universitários de medicina da Universidade Tiradentes e da Universidade Federal de Sergipe (Campos Lagarto), os quais de bom grado contribuíram com suas informações, indispensáveis para o desenvolvimento do projeto. À Universidade Tiradentes, pelo incentivo aos jovens universitários às novas descobertas na área da pesquisa através do programa de Bolsas de Iniciação Científica (PROBIC/UNIT), protocolo com número: 102.

\section{REFERÊNCIAS}

1. ALMEIDA AM, et al. Transtornos mentais comuns entre estudantes de medicina. J. bras. psiquiatr, 2007; 56(4):245251.

2. AMORIM BB, et al. Saúde mental do estudante de Medicina: psicopatologia, estresse, sono e qualidade de vida. Revista Psicologia, Diversidade e Saúde, 2018; 7(2):245-254.

3. BAPTISTA CA, et al. Social phobia in Brazilian university students: Prevalence, under-recognition and academic impairment in women. Journal of affective disorders, 2012; 136(3):857-861.

4. BEIDEL DC, et al. Social skills and social phobia: An investigation of DSM-IV subtypes. Behaviour research and therapy, 2010; 48(10):992-1001.

5. BRUNELLO N, et al. Social phobia: diagnosis and epidemiology, neurobiology and pharmacology, comorbidity and treatment. Journal of affective disorders, 2000; 60(2):61-74.

6. BURSTEIN M, et al. Social phobia and subtypes in the National Comorbidity Survey-Adolescent Supplement: prevalence, correlates, and comorbidity. Journal of the American Academy of Child \& Adolescent Psychiatry, 2011; 50(9):870-880.

7. COSTA EFO, et al. Transtornos mentais comuns entre estudantes de medicina da Universidade Federal de Sergipe: estudo transversal. Rev Bras Psiquiatr, 2010; 32(1):11-19.

8. ENG W, et al. Domains of life satisfaction in social anxiety disorder: Relation to symptoms and response to cognitivebehavioral therapy. Journal of Anxiety Disorders, 2005; 19(2):143-156.

9. FEHM L, et al. Size and burden of social phobia in Europe. European neuropsychopharmacology, 2015;15(4):453462. 
10. GIL AC. Métodos e técnicas de pesquisa social. 5a edição, SP, editora Atlas, 1999.

11. HEISER N, et al. Differentiating social phobia from shyness. Journal of anxiety disorders, 2009; 23(4):469-476.

12. MARAFANTI I, et al. Influência de sintomas ansiosos no desempenho acadêmico de formandos de medicina. Arquivos Médicos dos Hospitais e da Faculdade de Ciências Médicas da Santa Casa de São Paulo, 2013; $58(1): 18-23$.

13. OLIVEIRA MA, DUARTE AMM. Controle de respostas de ansiedade em universitários em situações de exposições orais. Revista Brasileira de Terapia Comportamental e Cognitiva, 2004; 6(2).

14. PEREIRA AS, et al. Déficits em habilidades sociais e ansiedade social: avaliação de estudantes de psicologia. Psicologia da Educação, 2014; 38:113-122.

15. PEREIRA SM, LOURENÇO LM. O estudo bibliométrico do transtorno de ansiedade social em universitários. Arquivos Brasileiros de Psicologia, 2012; 64(1).

16. REGIS JMO. Transtorno de ansiedade social e insatisfação com a imagem corporal em estudantes de medicina: prevalência e fatores associados (dissertação em Botucatu, SP): Universidade Estadual Paulista Júlio de Mesquita Filho, Faculdade de Medicina de Botucatu. 2015.

17. TENÓRIO LP, et al. Saúde mental de Estudantes de Escolas médicas com Diferentes modelos de Ensino medical. Revista Brasileira de educação Médica, 2016; 40(4):574-582.

18. WAGNER MF, et al. Sintomas de fobia social no ensino superior: uma amostra de população feminina. MudançasPsicologia da Saúde, 2014; 22(2):49-54.

19. WONG N, et al. Quality of life impairments among adults with social phobia: The impact of subtype. Journal of Anxiety Disorders, 2012; 26(1):50-57.

20. VIGNOCHI CM, et al. Considerações sobre aprendizagem baseada em problemas na educação em saúde. Revista HCPA, Porto Alegre, 2009; 29(1):45-50. 